\title{
Silent mutations in the phenylalanine hydroxylase gene as an aid to the diagnosis of phenylketonuria
}

\author{
L Kalaydjieva, B Dworniczak, C Aulehla-Scholz, M Devoto, G Romeo, M Sturhmann, \\ V Kucinskas, V Yurgelyavicius, J Horst
}

\begin{abstract}
Direct sequencing of the phenylalanine hydroxylase (PAH) gene indicated the existence of silent mutations in codons 232,245 , and 385 , linked to specific RFLP haplotypes in several Caucasian populations, namely Germans, Bulgarians, Italians, Turks, and Lithuanians. All three mutations create a new restriction site and can be easily detected on PCR amplified DNA. The usefulness of the silent mutations for diagnostic purposes depends on the haplotype distribution in the target population. The combined analysis of these markers and one or two PKU mutations forms a simple panel of diagnostic tests with full informativeness in a large proportion of PKU families, which helps to avoid the problems of genetic heterogeneity and of prenatal genomic Southern blot analysis.
\end{abstract}

Molecular diagnosis of phenylketonuria (PKU) became possible with the cloning and characterisation of the phenylalanine hydroxylase (PAH) gene ${ }^{1}$

Laboratory of Molecular Pathology, Institute of Obstetrics, Medical Academy, 2 Zdrave Street, Sofia 1431, Bulgaria.

L Kalaydjieva

Institut für Humangenetik, WWU, Münster, Germany.

B Dworniczak, C Aulehla-Scholz, J Horst

Laboratorio di Genetica Molecolare, Istituto G Gaslini, Genova, Italy.

M Devoto, G Romeo

Institut für Humangenetik, Freie Universität, Berlin, Germany.

M Stuhrmann

Institute for Maternal and Child Health, Vilnius, Lithuania.

V Kucinskas, V Yurgelyavicius

Correspondence to Dr Kalaydjieva.

Received for publication 17 December 1990

Revised version accepted for publication 6 March 1991. and the description of intragenic polymorphic restriction sites. ${ }^{2}$ RFLP analysis has been found to be informative for prenatal diagnosis in $70 \%$ to over $90 \%$ of at risk families in various European populations. ${ }^{3-6}$ However, compared to methods based on PCR amplified DNA, prenatal detection of Mendelian disorders by genomic Southern blot analysis has some well known limitations. Direct detection of mutation is a convenient diagnostic tool, but its feasibility is largely dependent on the extent of genetic heterogeneity of the disease in the target population. Sufficient information is now available to show the heterogeneous molecular basis of PKU, both in terms of numerous allelic mutations and interpopulation differences. Therefore, the molecular diagnosis of the disease is not an easy task and the increasing number of newly detected mutations adds to this complexity.

During a study of molecular defects leading to PKU in various Caucasian populations, we have identified three silent mutations in the PAH gene which are linked to specific polymorphic haplotypes in all ethnic groups investigated. Part of these findings has been published elsewhere. ${ }^{7}$ Here we discuss the possible use of these mutations as diagnostic markers for the prenatal detection of PKU.

Materials and methods

A total of 160 normal and mutant PAH alleles of German, Bulgarian, Italian, Turkish, and Lithuanian ethnic background were included in the study. RFLP analysis and haplotype assignment were as described previously. ${ }^{2}$ The cDNA probe phPAH247 was kindly provided by Dr S L C Woo.

Direct sequencing of exons 6,7 , and 11 of the PAH gene was performed on single stranded DNA templates obtained by asymmetrical amplification. ${ }^{8}$ Amplification and sequencing primers for exon 7 have been reported previously ${ }^{7}$ and those for exons 6 and 11 are shown in figs 1 and 2 .

Direct restriction analysis was performed after amplification of exons 6,7 , and 11 of the PAH gene. The restriction sites used were $D d e I$ for the detection of the silent mutation in exon 6, AluI for the mutation in exon 7 , and $M n l I$ for exon 11 . The 
$5^{\prime}$ - ggtcccogactccetctgctaacctaacctgcattctgctgtgccectgccetgct tgagacacctattttgtgcctgtattctagTGGGCAGCCCATCCCTCGAGTGGAATACA TGGAGGAAGAAAAGAAAACATGGGGCACAGTGTTCAAGACTCTGAAGTCCTTGTATAAAA CCCATGCTTGCTATGAGTACAATCACATTTTTCCACTTCTTGAAAAGTACTGTGGCTTCC ATGAAGATAACATTCCCCAGCTGGAAGACGTTTCTCAATTCCTGCAGAgtaagtccacat cagggtcaatggccetgcagaaagttgggggaggattgaggcagaggagagggaa - 3'
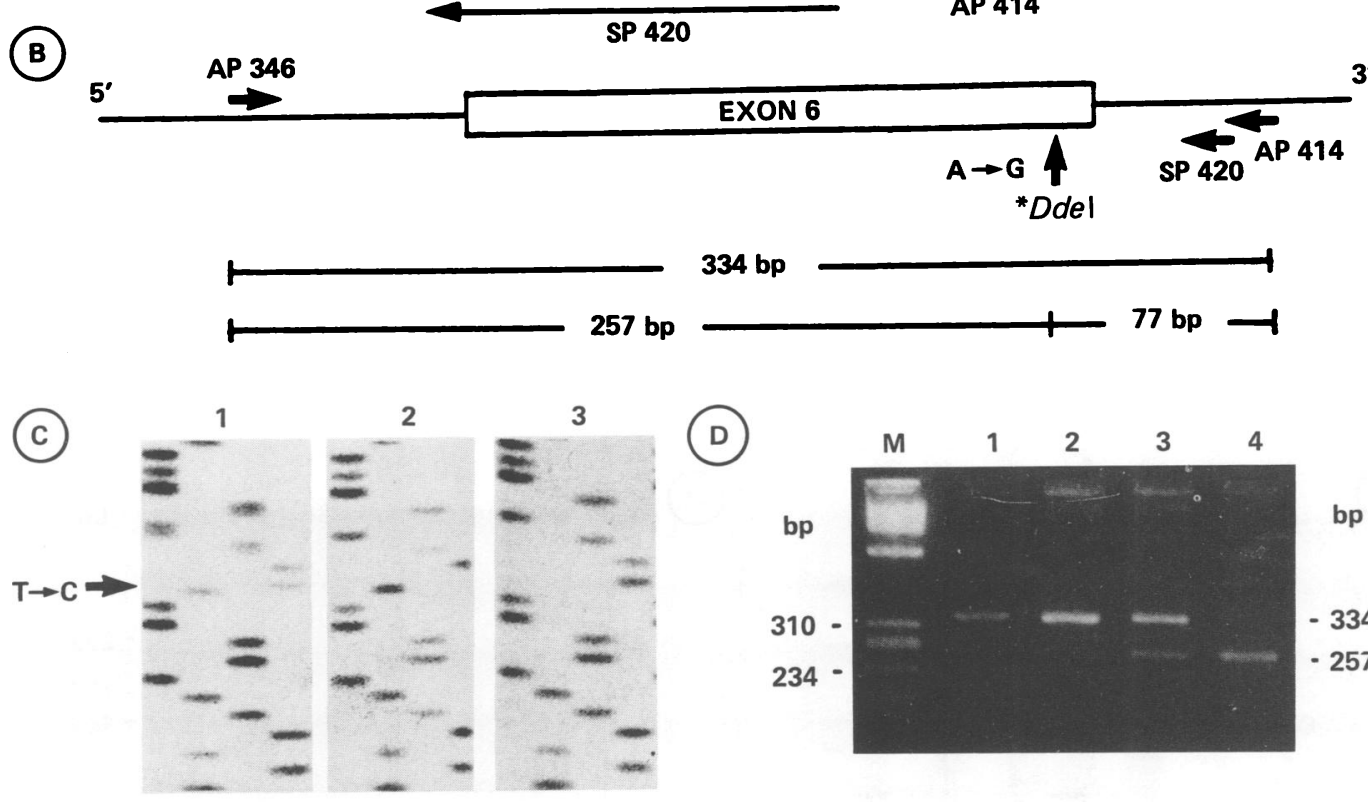

334 bp

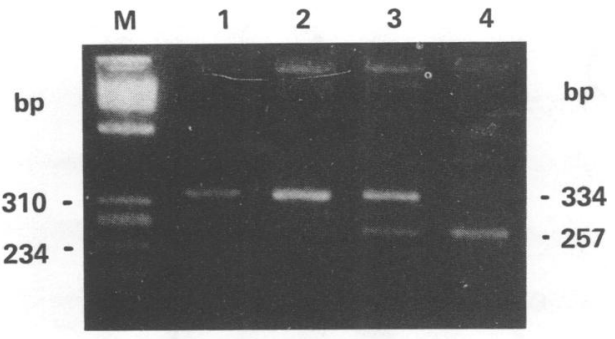

A C G T

Figure 1 ( $A$ ) Genomic sequence of exon 6 (capital letters) and the flanking introns of the PAH gene is shown. Arrows indicate the position and direction of amplification primers $(A P)$ and sequencing primer $(S P)$. (B) Schematic representation of exon 6; vertical arrow indicates the position of the $A \rightarrow G$ transition which creates a DdeI site. Below: sizes of the amplication product and DdeI cleavage products. (C) Sequences of the antisense strand of part of exon 6 . The silent mutation is indicated within the compound sequence ladders (in the antisense strand it appears as $T \rightarrow C$ transition). (1) Heterozygous type $(+-),(2,3)$ homozygous types $(++)$ and $(--)$ respectively. (D) DdeI digestion products of the amplified DNA fragments. Lane 1, undigested product; lanes 2 and 4, homozygous types (- ) and $(++)$ respectively; lane 3, heterozygous type (+-); lane M, size marker (HaeIII digested Phi X 174 DNA).

restriction fragments were electrophoretically separated on $4 \%$ 'NuSieve' agarose gels.

\section{Results}

MUTATION CHARACTERISTICS AND DETECTION An $A \rightarrow G$ transition was found in the third nucleotide of codon 232 of exon 6 . The new codon sequence (CAA $\rightarrow$ CAG) does not change the amino acid at position 232 of the protein molecule (Gln). The base substitution creates a $D d e I$ restriction site and digestion of the $334 \mathrm{bp}$ amplification product generates fragments which are $257 \mathrm{bp}$ and $77 \mathrm{bp}$ long (fig 1). A GTG $\rightarrow$ GTA (Val $\rightarrow \mathrm{Val}$ ) transition in codon 245, exon 7 , creates a new $A l u$ I restriction site. Identification and analysis have been described recently. ${ }^{7} \mathrm{~A} \mathrm{G} \rightarrow \mathrm{C}$ transversion in the last nucleotide of codon 385 of exon 11 changes the codon sequence from CTG to CTC (fig 2) without altering the encoded amino acid (Leu). The mutation creates an additional $M n l I$ restriction site. In the presence of the mutation, a $192 \mathrm{bp}$ long $\mathrm{MnlI}$ restriction fragment, which is part of the amplification product, is split into fragments $149 \mathrm{bp}$ and $43 \mathrm{bp}$ in length.

MENDELIAN INHERITANCE OF THE SILENT MUTATIONS The pattern of inheritance was checked by restriction analysis in at least one PKU family (parents and affected child) from each ethnic group. All three 
AP 255

(A) 5' - tgagagaaggggcacaaatggcctatgggatgcagcagggaatactgatcctgatt taacagtgataataacttttcacttggggcctacagTACTGCTTATCAGAGAAGCCAAAG CTTCTCCCCCTGGAGCTGGAGAAGACAGCCATCCAAAATTACACTGTCACGGAGTTCCAG CCCCTGTATTACGTGGCAGAGAGTTTTAATGATGCCAAGGAGAAAGTAAGgtgaggtggt gacaaaggtgagccactagctctgggggcctcctgactggtgccactcatctgtgggtgg SP 256

ttggccaggagagtggactccaatgtctacagcaactttgtaccatagacagttt - $\mathbf{3}^{\prime}$ AP 264
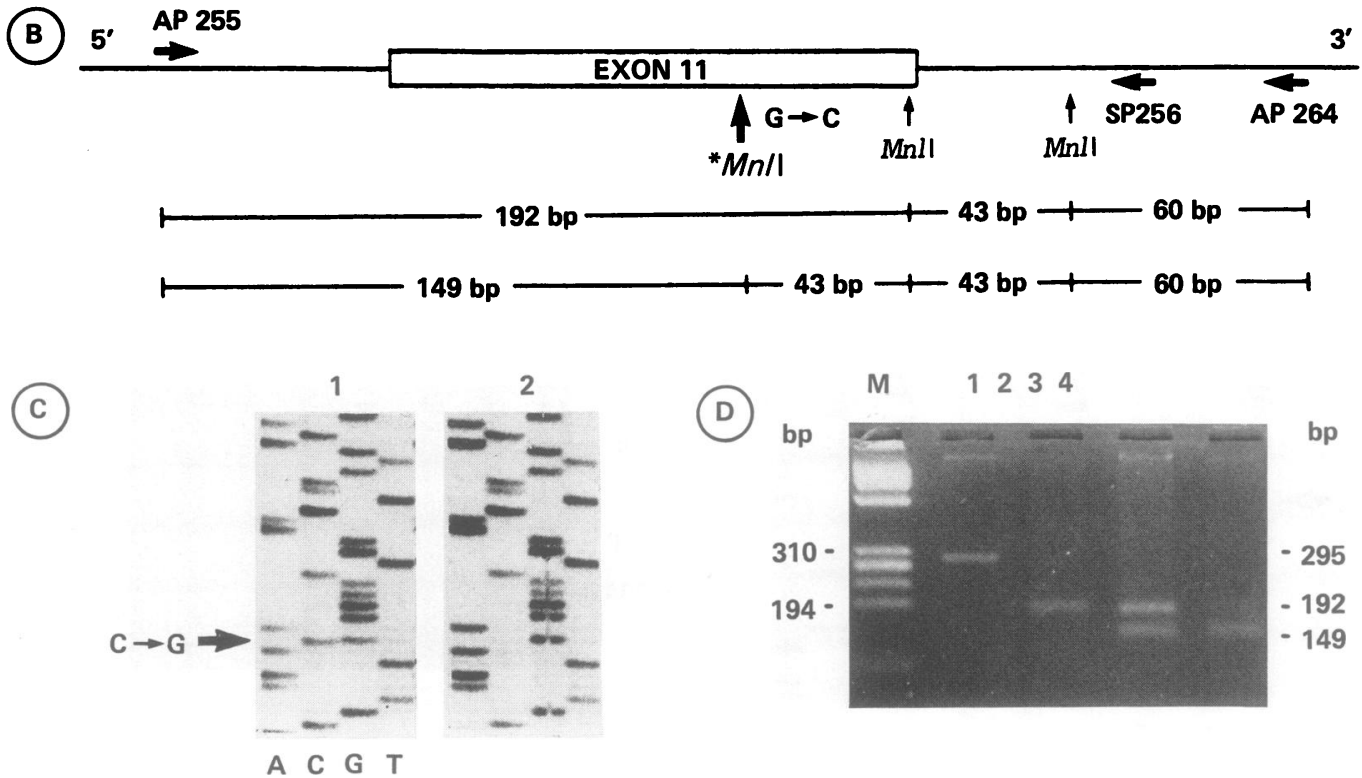

Figure 2 (A) Genomic sequence of exon 11 (capital letters) and flanking introns of the PAH gene is shown. Arrows indicate the position and direction of amplification primers $(A P)$ and sequencing primer $(S P)$. (B) Schematic representation of exon 11; vertical arrows indicate the positions of MnlI sites within the amplified product (asterisk indicates the polymorphic site introduced by the $G \rightarrow C$ transversion). Below: sizes of the amplification product and MnlI cleavage products. (C) Sequences of the antisense strand of part of exon 11. Arrow indicates the silent mutation within the compound sequence ladders (in the antisense strand it appears as $C \rightarrow G$ substitution). (1) Heterozygous type (+ -), (2) homozygous type $(++)$. (D) MnlI digestion products of the amplified DNA fragments electrophoretically separated on a $4 \%$ NuSieve agarose gel. Lane 1, undigested product; lanes 2 and 4, homozygous types (- - ) and ( + ) respectively; lane 3, heterozygous type (+-); lane M, size marker (HaeIII digested Phi X 174 DNA).

silent mutations were found to segregate as Mendelian traits.

\section{LINKAGE TO RFLP HAPLOTYPES}

The presence of the silent mutations was tested on normal and mutant PAH alleles which belong to the main polymorphic haplotypes found in European populations (table 1). The base substitution in codon 232 of exon 6 was detected on all haplotype 3, 4 , and 7 alleles. The association was present in all ethnic groups studied. It was independent of the presence or absence of a PKU mutation on the same allele and of the nature of the mutation in the case of PKU alleles.

The silent mutation in codon 245 , exon 7 , was previously reported to be linked with haplotype 4 (normal and mutant) and haplotypes 16, 17, 28, and 3 (normal) in the German population. ${ }^{7}$ In the present study of different ethnic groups, the transition in codon 245 was not detected on normal haplotype 3 alleles of Bulgarian, Turkish, or Italian ethnic background. It was invariably present on all haplotype 4 chromosomes and also on the few normal haplotype 16,17 , and 28 alleles which were available 
Table 1 Main haplotypes linked to the silent mutations in the PAH gene*.

\begin{tabular}{|c|c|c|c|c|c|c|}
\hline \multirow[b]{3}{*}{ Haplotype } & \multicolumn{6}{|c|}{ Silent mutations } \\
\hline & \multicolumn{2}{|c|}{ Exon 6} & \multicolumn{2}{|c|}{ Exon 7} & \multicolumn{2}{|c|}{ Exon 11} \\
\hline & Tested & Positive & Tested & Positive & Tested & Positive \\
\hline $\begin{array}{l}1 \\
2 \\
3 \\
4 \\
5 \\
6 \\
7\end{array}$ & $\begin{array}{r}17 \\
16 \\
11 \\
34 \\
4 \\
11 \\
9\end{array}$ & $\begin{array}{r}0 \\
0 \\
11 \\
34 \\
0 \\
0 \\
9\end{array}$ & $\begin{array}{r}18 \\
9 \\
7 \\
22 \\
4 \\
14 \\
7\end{array}$ & $\begin{array}{r}0 \\
0 \\
0 \\
22 \\
0 \\
0 \\
0\end{array}$ & $\begin{array}{r}14 \\
12 \\
12 \\
12 \\
5 \\
16 \\
14\end{array}$ & $\begin{array}{r}0 \\
0 \\
12 \\
0 \\
0 \\
0 \\
14\end{array}$ \\
\hline
\end{tabular}

* The table includes alleles tested by restriction analysis.

for analysis. The silent mutation in exon 11 was detected on all haplotype 3 and 7 alleles, normal and mutant, in the ethnic groups studied.

\section{DIAGNOSTIC APPLICATION}

The diagnostic use of the silent mutations in the PAH gene is illustrated by the pedigree shown in fig 3. Both mutant alleles, haplotypes 4 and 7 , carry an unknown mutation. The maternal normal allele belongs to haplotype 1 , so therefore the mother is fully informative for the silent mutations linked to haplotype 4 (the DdeI site in exon 6 and the AluI site in exon 7). The paternal alleles (HT 7 mutant, HT 4 normal) can be distinguished by $\mathrm{MnlI}$ restriction of exon 11.

USEFULNESS IN DIFFERENT POPULATIONS

The diagnostic information gained by the analysis of the silent mutations alone was tested in the German and the Bulgarian groups of PKU families (table 2). In the PKU families from Germany, the analysis was found to be fully informative in $20 \%$ and half informative in $48 \%$ of the couples. In the Bulgarian group, only $9 \%$ were fully informative and $65 \%$ were half informative.

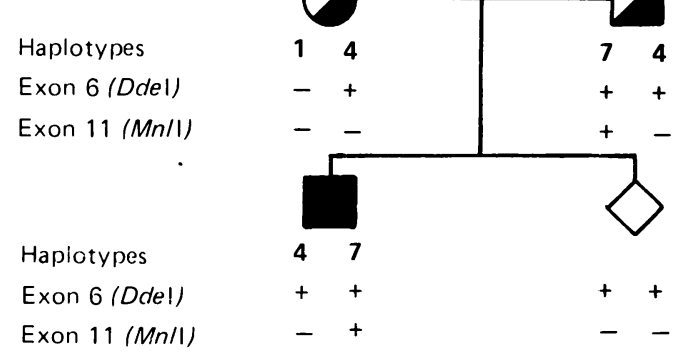

Figure 3 Pedigree showing the diagnostic use of the silent mutations in the PAH gene.
The different informativeness is the result of the differences in haplotype distribution in the two populations. In PKU families originating from the western part of Germany, haplotype 3 has been reported to account for $11 \%$ of mutant PAH alleles, ${ }^{4}$ whereas in eastern and southern European populations it is virtually absent. ${ }^{56}$ In addition, haplotype 4 has a higher frequency on both normal and mutant alleles in Germany than in Bulgaria. ${ }^{46}$

DESIGNING A MINIMUM DIAGNOSTIC PANEL

Regardless of molecular heterogeneity, one, or the major, PKU mutation has been reported to account for a large proportion of mutant alleles in most Caucasian populations studied. We have therefore estimated the informativeness of a minimum panel of diagnostic tests, including the silent mutations in the PAH gene and two PKU mutations, in the ethnic groups investigated. The amino acid substitution in codon $408^{8}$ and a newly detected frequent splicing defect in the PAH gene ${ }^{9}$ were selected on the basis of previously reported findings concerning the predominant PKU mutations in these populations. ${ }^{461011}$ The data on the combined use of the silent mutations together with the most common PKU defects are presented in table 3.

This approach makes prenatal diagnosis of PKU fully informative in $73 \%$ of Lithuanian, $62 \%$ of Bulgarian, and $46 \%$ of West German PKU families. The proportion of uninformative families in Bulgaria and Lithuania is reduced to 7 to $8 \%$. Informativeness is lower in populations where haplotype 1 is

Table 2 Informativeness of the silent mutations in two groups of PKU families of different ethnic background.

\begin{tabular}{lccc}
\hline & \multicolumn{3}{c}{ Informativeness of the silent mutation } \\
\cline { 2 - 4 } Ethnic origin & $100 \%$ & $50 \%$ & $0 \%$ \\
\hline $\begin{array}{c}\text { German } \\
(\mathrm{n}=46)\end{array}$ & $9(20 \%)$ & $22(48 \%)$ & $15(32 \%)$ \\
$\begin{array}{c}\text { Bulgarian } \\
(\mathrm{n}=23)\end{array}$ & $2(9 \%)$ & $15(65 \%)$ & $6(26 \%)$ \\
\hline
\end{tabular}


Table 3 Combined informativeness of silent mutations and two frequent PKU mutations (Arg ${ }^{408} \rightarrow \operatorname{Tr} \mathrm{p}^{408}$, splice mutation in intron 10) in several Caucasian populations.*

\begin{tabular}{lccc}
\hline & \multicolumn{3}{c}{ Informativeness for prenatal diagnosis } \\
\cline { 2 - 4 } Ethnic group & $100 \%$ & $50 \%$ & $0 \%$ \\
\hline $\begin{array}{c}\text { Lithuanian } \\
(\mathrm{n}=26)\end{array}$ & $19(73 \%)$ & $5(19 \%)$ & $2(8 \%)$ \\
$\begin{array}{c}\text { Bulgarian } \\
(\mathrm{n}=29)\end{array}$ & $18(62 \%)$ & $9(31 \%)$ & $2(7 \%)$ \\
$\begin{array}{c}\text { German } \\
(\mathrm{n}=46)\end{array}$ & $21(46 \%)$ & $14(30 \%)$ & $11(24 \%)$ \\
$\begin{array}{c}\text { Italian } \\
(\mathrm{n}=19)\end{array}$ & $8(42 \%)$ & $6(32 \%)$ & $5(26 \%)$ \\
$\begin{array}{c}\text { Turkish } \\
(\mathrm{n}=15)\end{array}$ & $6(40 \%)$ & $5(33 \%)$ & $4(27 \%)$ \\
\hline
\end{tabular}

* Estimates of informativeness are based on previously published haplotype and mutation distribution data 41011 and on linkage between the silent mutations and specific haplotypes established in the present study.

very common, such as the Italian ${ }^{5}$ and Turkish $^{11}$ populations, and is likely to remain low because of genetic heterogeneity and the large number of PKU mutations linked to this haplotype. ${ }^{12-14}$

\section{Discussion}

The existence of the silent mutations in the PAH gene has to be taken into account when methods for the non-specific differentiation between normal and mutant alleles (for example, GC clamping or SSCP) are used for diagnostic purposes. The isolated diagnostic use of the three silent mutations is of limited value and allows fully informative genotyping in fewer than $20 \%$ of PKU families. Because of the tight linkage with specific polymorphic haplotypes, the usefulness of these mutations in different ethnic groups depends on haplotype distribution and is greater in populations with a high frequency of haplotypes 3, 4, and 7 . In such populations the analysis of the silent mutations has the additional advantage of getting round the problem of the heterogeneity of PKU defects linked to haplotypes 4 and 7. When combined with testing for the prevalent PKU molecular defects, the silent mutations in the PAH gene form a simple panel of diagnostic tests, which are fully informative in a significant proportion of PKU families. This limits further investigation to two or three polymorphic sites in the remaining parts of families at risk.

This study was supported by the Deutsche Forschungsgemeinschaft, the Bundesministerium fuer Forschung und Technologie, and the Bulgarian Neurosciences and Behaviour Research Programme. LK was a fellow of the Alexander von Humboldt Foundation.

1 Kwok S, Ledley FD, Robson JH, Woo SLC. Nucleotide sequence of a full-length $\mathrm{CDNA}$ clone of human phenylalanine hydroxylase. Biochemistry 1985;24:556-61.

2 Lidsky AS, Ledley FD, DiLella AG, et al. Extensive restriction site polymorphism at the human phenylalanine hydroxylase locus and application in prenatal diagnosis of phenylketonuria. Am f Hum Genet 1985;37:619-34.

3 Daiger S, Chakraborty R, Reed L, et al. Polymorphic DNA haplotypes at the phenylalanine hydroxylase (PAH) locus in European families with phenylketonuria (PKU). Am f Hum Genet 1989;45:310-18.

4 Aulehla-Scholz C, Vorgert M, Sautter E, et al. Phenylketonuria: a distribution of DNA diagnostic patterns in German families. Hum Genet 1988;78:353-5.

5 Dianzani I, Devoto M, Camaschella C, et al. Haplotype distribution and molecular defects at the phenylalanine hydroxylase locus in Italy. Hum Genet 1990;86:69-72.

6 Kalaydjieva L, Dworniczak B, Aulehla-Scholz C, Kremensky I, Bronzova J, Horst J. Classical phenylketonuria in Bulgaria: RFLP haplotypes and frequency of the major mutations. $f$ Med Genet 1990;27:742-5.

7 Dworniczak B, Aulehla-Scholz C, Horst J. Phenylalanine hydroxylase gene: silent mutation uncovers evolutionary origin of different alleles. Clin Genet 1990;38:270-3.

8 Spiegelberg R, Aulehla-Scholz C, Erlich H, Horst J. A betathalassemia gene caused by a 290-base pair deletion: analysis by direct sequencing of enzymatically amplified DNA. Blood 1989;73:1695-8.

9 Dworniczak B, Kalaydjieva L, Aulehla-Scholz C, Bartholomé $\mathrm{K}$, Grudda $\mathrm{K}$, Horst J. Aberrant splicing of phenylalanine hydroxylase mRNA: the major cause for phenylketonuria in parts of southern Europe. Genomics (in press).

10 Kalaydjieva L, Dworniczak B, Kucinskas V, Yurgeliavicius V, Kunert E, Horst J. Geographical distribution gradients of the major PKU mutations and the linked haplotypes. Hum Genet 1991;86:411-3.

11 Lichter-Konecki U, Schlotter M, Yaylak C, et al. DNA haplotype analysis at the phenylalanine hydroxylase locus in the Turkish population. Hum Genet 1989;81:373-6.

12 Okano Y, Wang T, Eisensmith RC, et al. Missense mutations associated with RFLP haplotypes 1 and 4 of the human phenylalanine hydroxylase gene. Am $f$ Hum Genet 1990;46:18-25.

13 Dworniczak B, Grudda K, Stümper J, Bartholomé K, AulehlaScholz C, Horst J. Phenylalanine hydroxylase gene: novel missense mutation in exon 7 causing severe phenylketonuria. Genomics 1991;9:193-9.

14 Kalaydjieva L, Dworniczak B, Kremensky L, et al. Heterogeneity of mutations in Bulgarian phenylketonuria haplotype 1 and 4 alleles. Clin Genet (in press). 